\title{
Calibration of piezoelectric RL shunts with explicit residual mode correction
}

\author{
Høgsberg, Jan Becker; Krenk, Steen
}

Published in:

Journal of Sound and Vibration

Link to article, DOI:

10.1016/j.jsv.2016.08.028

Publication date:

2017

Document Version

Peer reviewed version

Link back to DTU Orbit

Citation (APA):

Høgsberg, J. B., \& Krenk, S. (2017). Calibration of piezoelectric $R L$ shunts with explicit residual mode correction. Journal of Sound and Vibration, 386, 65-81. https://doi.org/10.1016/j.jsv.2016.08.028

\section{General rights}

Copyright and moral rights for the publications made accessible in the public portal are retained by the authors and/or other copyright owners and it is a condition of accessing publications that users recognise and abide by the legal requirements associated with these rights.

- Users may download and print one copy of any publication from the public portal for the purpose of private study or research.

- You may not further distribute the material or use it for any profit-making activity or commercial gain

- You may freely distribute the URL identifying the publication in the public portal

If you believe that this document breaches copyright please contact us providing details, and we will remove access to the work immediately and investigate your claim. 


\title{
Calibration of piezoelectric $R L$ shunts with explicit residual mode correction
}

\author{
Jan Høgsberg ${ }^{1}$ and Steen Krenk \\ Department of Mechanical Engineering, \\ Technical University of Denmark, DK-2800 Kongens Lyngby, Denmark \\ jhg@mek.dtu.dk , sk@mek.dtu.dk
}

\begin{abstract}
Piezoelectric $R L$ (resistive-inductive) shunts are passive resonant devices used for damping of dominant vibration modes of a flexible structure and their efficiency relies on the precise calibration of the shunt components. In the present paper improved calibration accuracy is attained by an extension of the local piezoelectric transducer displacement by two additional terms, representing the flexibility and inertia contributions from the residual vibration modes not directly addressed by the shunt damping. This results in an augmented dynamic model for the targeted resonant vibration mode, in which the residual contributions, represented by two correction factors, modify both the apparent transducer capacitance and the shunt circuit impedance. Explicit expressions for the correction of the shunt circuit inductance and resistance are presented in a form that is generally applicable to calibration formulae derived on the basis of an assumed single-mode structure, where modal interaction has been neglected. A design procedure is devised and subsequently verified by a numerical example, which demonstrates that effective mitigation can be obtained for an arbitrary vibration mode when the residual mode correction is included in the calibration of the $R L$ shunt.
\end{abstract}

Keywords: $R L$ shunt circuit, Piezoelectric damping, Structural dynamics, Shunt calibration, Residual mode correction

\section{Introduction}

Piezoelectric transducers are installed to increase the attainable level of damping in many different types of engineering structures, thereby improving the overall vibration response properties. Because of their electromechanical coupling, piezoelectric transducers may be employed as sensor/actuator pairs in active feedback vibration control [1] as well as passively by shunting with supplemental electronic circuits [2]. The concept of resonant piezoelectric $R L$ shunt damping was originally proposed by Forward [3], while current research commonly refers to the work by

\footnotetext{
${ }^{1}$ Corresponding author: jhg@mek.dtu.dk
} 
Hagood and von Flotow [4] concerning the series $R L$ circuit and Wu [5] for the corresponding parallel configuration.

The efficiency of resonant $R L$ shunt damping depends primarily on the precise synchronization of the electric circuit frequency, governed by the inductor $L$, with the resonance frequency of the structure, while the corresponding damping component, represented by the resistor $R$, must dissipate sufficient energy, [6]. The tuning of resonant dampers dates back to the analysis of the mechanical tuned mass damper [7], for which the classic frequency response calibration based on identical amplitudes at two neutral frequencies implies equal damping of the two modes associated with the targeted vibration form, [8]. As demonstrated in [4] this desirable equivalence between the neutral point principle and equal modal damping is lost for piezoelectric $R L$ shunt damping, whereby the robustness of having identical damping in the targeted modes is not obtained when tuning the shunt circuit components with respect to minimum vibration amplitudes. Thus, the development of specific calibration strategies for $L$ and $R$ based on different objectives and optimality criteria has been - and still is - an active field of research, for example concerning damping of multiple vibration modes [9], shunt circuits with supplemental capacitance [10], tuning with respect to different types of motion [11], design by mechanical analogies [12] and calibration based on the $H_{\infty}$-norm of the response amplitude [13]. Furthermore, a balanced calibration principle in [14] determines the inductance $L$ based on the equal damping property like for the tuned mass damper [8], while the resistance $R$ is subsequently chosen to secure a flat plateau of the dynamic amplification curve around the resonance frequency. As demonstrated by a numerical example in Section 5 this balanced principle constitutes an appropriate compromise between large modal damping and effective response reduction.

Piezoelectric transducers are commonly placed locally on a flexible structure, thereby activating residual modes not specifically addressed by the shunt circuit damping. This energy spill-over naturally alters the closed-loop dynamics of the structure, as discussed in [15], whereby the performance deteriorates when using calibration principles based on an assumed single-mode response without any modal interaction. The influence of residual modes can to some extent be accounted for by including the quasi-static flexibility from the modes with higher frequency, as demonstrated in $[16,17]$. In dynamic analysis of discretized structures reduced order models can be obtained by a quasi-static representation of the high-frequency modes by subtracting the flexibility of the dynamic modes from the inverse of the stiffness matrix, [18]. This type of quasi-static representation of the residual mode contributions has been adopted for calibration of the mechanical tuned mass damper in [19] and subsequently for piezoelectric $R L$ shunt damping in [20]. In both cases explicit calibration formulae have been derived and the accuracy has been verified by numerical examples concerning the damping of the lowest vibration form, thus with energy spill-over arising only from modes with frequencies above the targeted resonance. 
However, in vibration problems commonly addressed by piezoelectric $R L$ shunts the largest energy content can occur in intermediate vibration modes, as for example in the case of acoustic vibrations of membranes [3] and plates [15,21]. In the corresponding calibration procedures a more refined residual mode correction may be needed, compensating for non-resonant modes both below and above the targeted resonance. Recently, a consistent correction format has been proposed by Krenk and Høgsberg [22], where both flexibility and inertia contributions from the non-resonant modes have been represented, thereby extending the quasi-static representation in [19, 20]. It is demonstrated in [22] that the inertia correction is of importance for damping of intermediate modes by tuned mass dampers, acting on the absolute motion of the structure, whereas for inerter-based devices attached between two points in the structure the residual mode flexibility constitutes the most important correction.

In the present paper the consistent residual mode correction with both flexibility and inertia contributions is developed in the context of $R L$ shunt damping. It is demonstrated that general expressions for the correction of the shunt circuit inductor and resistor can be obtained in explicit form, making the present approach generic and thus applicable to a large variety of calibration formulae and principles derived on the basis of a simplified single-mode structure without the modal interaction inherently present in dynamics of flexible structures. In reduced order models the attainable damping is governed by the generalized electromechanical coupling coefficient, which is a rather complex quantity as it comprises the specific characteristics of the transducer, its location and placement on the structure and the structural modal properties, see [23]. Thus, it is commonly estimated either numerically $[24,25]$ or experimentally [26] in terms of the two natural frequencies of the structure associated with short and open circuit transducer electrodes. It is demonstrated that the proposed residual mode corrections also secure equal modal damping when the shunt calibration is based on this estimate instead of the exact coupling coefficient.

\section{Structure with piezoelectric transducer}

The purpose of mounting piezoelectric $R L$ (resistive-inductive) shunts on a structure is to increase the damping of a resonant vibration mode of the structure. This requires attention to the location of the transducer on the structure as well as appropriate calibration of the transducer based on the combined transducer-structure system. The governing equation of motion of the vibrating structure can be written in discretized form as

$$
\left(-\omega^{2} \mathbf{M}+\mathbf{K}\right) \mathbf{u}+\mathbf{w} f=\mathbf{f}_{e}
$$

where $\mathbf{M}$ and $\mathbf{K}$ are the mass and stiffness matrix of the structure, while the complex-valued harmonic response is represented with time factor $\exp (i \omega t)$ via the complex amplitudes $\mathbf{u}$. The corresponding external load components are contained in $\mathbf{f}_{e}$, while $f$ represents the amplitude of 
(a)

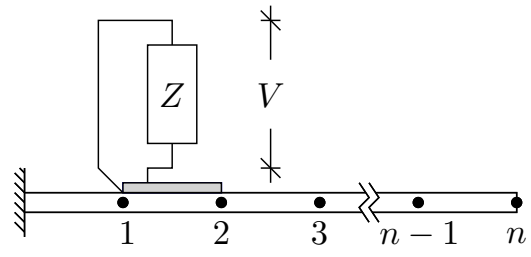

(b)

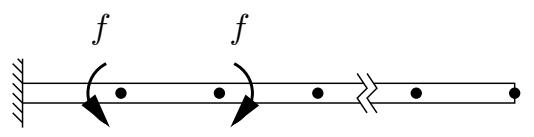

Figure 1: Discretized beam with (a) shunted piezoelectric transducer and (b) moment pair $f$.

the force exerted by the piezoelectric transducer on the structure through the connectivity vector w.

\subsection{Connectivity}

The attainable level of damping and the shunt circuit calibration depends very much on the attachment and location of the piezoelectric transducer on the vibrating host structure. A typical benchmark problem is illustrated in Fig. 1(a), where a laminate type transducer is placed between nodes 1 and 2 of a discretized cantilever beam. In this case the connectivity vector can be formulated as $\mathbf{w}=[0,1,0,-1,0, \ldots]^{\mathrm{T}}$, with plus/minus unit entries associated with the two rotational degrees of freedom of the finite beam element. When neglecting the small axial force component, the transducer $f$ represents the nodal bending moment couple shown in Fig. 1(b), while the collocated transducer displacement

$$
u=\mathbf{w}^{\mathrm{T}} \mathbf{u}
$$

represents the corresponding change in cross section rotation between the nodes of attachment. Alternatively, the connectivity vector $\mathbf{w}$ may contain the transverse distance between the neutral axes of beam and laminate transducer, instead of the unit values, in which case $f$ represents the axial transducer force, while $u$ is the corresponding transducer elongation. In both cases the generalized force $f$ produces work through the corresponding generalized displacement $u$.

In structural control applications the piezoelectric transducer forces commonly act locally on the vibrating structure, whereby the displacement $u$ inherently contains contributions from multiple vibration modes, including modal spill-over from non-resonant vibration forms not targeted by the shunt damping. This spill-over effect may lead to substantial detuning of the shunt circuit components and consequently deterioration of the damping performance. In the present paper a tuning principle is derived for resonant $R L$ shunts, in which the simple calibration formulae for resistance $R$ and inductance $L$ for an ideal single-mode structure are modified in explicit form to consistently account for the interaction with the non-resonant residual vibration modes of the structure. 
(a)

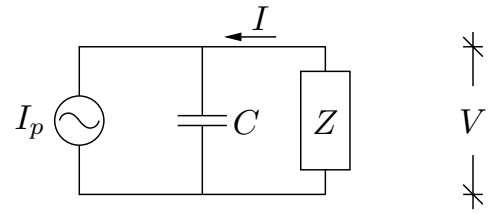

(b)

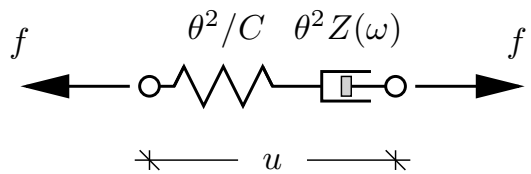

Figure 2: (a) Piezoelectric transducer connected to electronic shunt circuit and (b) equivalent mechanical model.

\subsection{Electromechanical coupling}

For piezoelectric transducers the supplemental force $f$ in Eq. (1) is proportional to the voltage $V$ across the transducer electrodes, as illustrated in Fig. 1(a). It can therefore be written as

$$
f=\theta V
$$

where $\theta$ is the electromechanical coupling coefficient of the particular transducer configuration. Because the force is proportional to voltage the stiffness matrix $\mathbf{K}$ in Eq. (1) governs the natural frequencies of the structure associated with short-circuited transducer electrodes $(V=0)$.

For piezoelectric transducers the electromechanical coupling effect is represented by the balance or sensor equation $[27,28]$

$$
Q=-\theta u+C V
$$

where $Q$ is the charge produced by the transducer, with contributions from the mechanical deformation $(-\theta u)$ and from the capacitative effect $(C V)$ with capacitance $C$ associated with constrained transducer boundaries $(u=0)$.

The electronic circuit representation of Eq. (4) is shown in Fig. 2(a), in which the current source $I_{p}=\mathrm{i} \omega \theta u$ represents the electrical displacement produced by the harmonic transducer motion $u$, with the electromechanical coupling coefficient $\theta$ uniquely defined by Eqs. (1)-(4). The current source representation in Fig. 2(a) implies that the corresponding transducer capacitance $C$ is placed in parallel. This representation is effectively used in Section 3.2 to combine $C$ with the equivalent impedance of the mechanical flexibility from the residual vibration modes.

\subsection{Shunt damping}

As indicated in Fig. 1(a) the electric loading on the vibrating structure is produced by a shunt circuit with frequency dependent impedance $Z(\omega)$, governing the relation

$$
V=Z(\omega) I=-\mathrm{i} \omega Z(\omega) Q
$$


between voltage and charge, where $I=-\mathrm{i} \omega Q$ is the current in the transducer electrodes, as shown in Fig. 2(a). Elimination of $Q$ between Eqs. (4) and (5) gives a relation between voltage $V$ and displacement $u$, which is then substituted into Eq. (3) to obtain the equivalent force-displacement relation

$$
u=\left(\frac{C}{\theta^{2}}+\frac{1}{\mathrm{i} \omega \theta^{2} Z(\omega)}\right) f
$$

This force-displacement relation with additive displacement contributions corresponds to an equivalent Maxwell-type mechanical model, shown in Fig. 2(b), with equivalent spring stiffness $\theta^{2} / C$ and a viscous dashpot with frequency dependent viscous coefficient $\theta^{2} Z(\omega)$. Thus, for a pure resistive shunt with constant impedance $Z(\omega)=R$ the dashpot represents a viscous damper, whereby the mechanical model in Fig. 2(b) recovers a pure Maxwell element. However, if the impedance represents a supplemental capacitance $Z(\omega)=1 /\left(\mathrm{i} \omega C_{*}\right)$ the dashpot is transformed into an elastic element and the mechanical model therefore appears as a spring with combined stiffness $\theta^{2} /\left(C+C_{*}\right)$. Finally, for an inductance with $Z(\omega)=\mathrm{i} \omega L$ the dashpot in Fig. 2(b) represents inertia by a so-called inerter device with inertance $\theta^{2} L$, see $[29,30]$. The mechanical analogy in Fig. 2(b) demonstrates that placing electronic components in parallel in the shunt circuit corresponds to an equivalent mechanical series connection because $f$ and $V$ represent mechanical and electrical forcing, respectively.

\section{Modal equations}

The electronic components of a resonant $R L$ shunt circuit are calibrated with respect to a single dominant vibration mode of the structure, in the following identified by a subscript $r$. The mode shape vector $\mathbf{u}_{j}$ and the natural angular frequency $\omega_{j}$ of the vibrating structure are determined by the eigenvalue problem associated with Eq. (1),

$$
\left(\mathbf{K}-\omega_{j}^{2} \mathbf{M}\right) \mathbf{u}_{j}=\mathbf{0}
$$

The modal expansion with respect to the normalized mode shape vector $\mathbf{u}_{j} /\left(\mathbf{w}^{\mathrm{T}} \mathbf{u}_{j}\right)$ is

$$
\mathbf{u}=\sum_{j} \frac{\mathbf{u}_{j}}{\mathbf{w}^{\mathrm{T}} \mathbf{u}_{j}} u_{j}
$$

with modal amplitude $u_{j}$. Substitution into Eq. (1) followed by pre-multiplication with the normalized mode shape vector gives the scalar modal equation of motion

$$
\left(-\omega^{2} m_{j}+k_{j}\right) u_{j}+f=f_{j}
$$

with modal mass $m_{j}$, modal stiffness $k_{j}$ and modal load $f_{j}$ given as

$$
m_{j}=\frac{\mathbf{u}_{j}^{\mathrm{T}} \mathbf{M} \mathbf{u}_{j}}{\left(\mathbf{w}^{\mathrm{T}} \mathbf{u}_{j}\right)^{2}} \quad, \quad k_{j}=\frac{\mathbf{u}_{j}^{\mathrm{T}} \mathbf{K} \mathbf{u}_{j}}{\left(\mathbf{w}^{\mathrm{T}} \mathbf{u}_{j}\right)^{2}} \quad, \quad f_{j}=\frac{\mathbf{u}_{j}^{\mathrm{T}} \mathbf{f}_{e}}{\mathbf{w}^{\mathrm{T}} \mathbf{u}_{j}}
$$

while the modal transducer force $f$ appears explicitly in Eq. (9) because of the normalization of the mode shape vectors in Eq. (8) to unity across the transducer connection. 


\subsection{Residual flexibility and inertia}

In Eq. (2) the displacement $u$ contains contributions from both the targeted resonant vibration form $(j=r)$ and other residual, and thereby non-resonant, modes $(j \neq r)$. As recently demonstrated in [22] the influence of the interaction with the non-resonant modes can be represented by the extended modal representation

$$
u=u_{r}-\left(\frac{1}{k_{r}^{\prime}}-\frac{1}{k_{r}^{\prime \prime}} \frac{\omega_{r}^{2}}{\omega^{2}}\right) f
$$

where $u_{r}$ is the modal coordinate of the resonant mode, while the parenthesis contains a flexibility and an inertia correction term due to contributions from the residual modes. Thus, the apparent displacement of the resonant mode can be represented as

$$
u_{r}=u+u_{r}^{\prime}+u_{r}^{\prime \prime}
$$

where the added displacement amplitudes $u_{r}^{\prime}$ and $u_{r}^{\prime \prime}$ from residual mode flexibility and inertia, respectively, follow from Eq. (11) as

$$
u_{r}^{\prime}=\frac{f}{k_{r}^{\prime}} \quad, \quad u_{r}^{\prime \prime}=-\frac{\omega_{r}^{2}}{\omega^{2}} \frac{f}{k_{r}^{\prime \prime}}
$$

Expressions for the modal correction parameters $k_{r}^{\prime}$ and $k_{r}^{\prime \prime}$ in (13) have been derived in [22] by matching the approximate and the full frequency response representations as well as their derivatives with respect to the squared frequency $\omega^{2}$ at the resonance frequency $\omega_{r}$.

The flexibility and inertia coefficients $k_{r}^{\prime}$ and $k_{r}^{\prime \prime}$ represent contributions from the non-resonant modes. Following [22], the first step is to introduce a mass matrix $\mathbf{M}_{r}$, in which the contribution from the resonant mode has been removed,

$$
\mathbf{M}_{r}=\mathbf{M}-\frac{\left(\mathbf{M} \mathbf{u}_{r}\right)\left(\mathbf{M} \mathbf{u}_{r}\right)^{\mathrm{T}}}{\mathbf{u}_{r}^{\mathrm{T}} \mathbf{M} \mathbf{u}_{r}}
$$

Using this mass matrix a frequency shifted form $\mathbf{K}_{r}$ of the stiffness matrix is then introduced as

$$
\mathbf{K}_{r}=\mathbf{K}-\omega_{r}^{2} \mathbf{M}_{r}
$$

This matrix is regular, as the response to the resonant mode corresponds to the original static response, while the response to the non-resonant modes has a non-singular frequency shift. Thus, the matrix $\mathbf{K}_{r}^{-1}$ can be found by straightforward inversion of $\mathbf{K}_{r}$. The corresponding expressions are conveniently written in the format

$$
\frac{1}{k_{r}^{\prime}}=\mathbf{w}^{\mathrm{T}} \mathbf{K}_{r}^{-1} \mathbf{w}-\frac{1}{k_{r}}+\frac{1}{k_{r}^{\prime \prime}}
$$

and

$$
\frac{1}{k_{r}^{\prime \prime}}=\left(\mathbf{w}^{\mathrm{T}} \mathbf{K}_{r}^{-1} \mathbf{K} \mathbf{K}_{r}^{-1} \mathbf{w}\right)-\left(\mathbf{w}^{\mathrm{T}} \mathbf{K}_{r}^{-1} \mathbf{w}\right)
$$


The first formula (16) defines the background flexibility $1 / k_{r}^{\prime}$ as the flexibility associated with the modified stiffness matrix $\mathbf{K}_{r}$ with subtraction of the modal flexibility $1 / k_{r}$ and addition of the equivalent inertia flexibility $1 / k_{r}^{\prime \prime}$. In Eq. (17) the equivalent background inertia flexibility $1 / k_{r}^{\prime \prime}$ is defined by combining the modified flexibility from $\mathbf{K}_{r}^{-1}$ with that of a higher order matrix product $\mathbf{K}_{r}^{-1} \mathbf{K K}_{r}^{-1}$. As demonstrated in [22] both flexibilities $1 / k_{r}^{\prime}$ and $1 / k_{r}^{\prime \prime}$ are non-negative.

A quasi-static representation containing only a flexibility correction is often used in connection with truncated modal response analysis, and has been used in [20] for calibration of $R L$ shunt circuits for damping of the lowest vibration mode of a flexible structure. This representation can be recovered from the expressions above by replacing the modified stiffness matrix $\mathbf{K}_{r}$ by the original stiffness matrix $\mathbf{K}$. Hereby the equivalent background inertia flexibility vanishes, $1 / k_{r}^{\prime \prime}=0$, and the expression of the background stiffness coefficient takes the simplified form

$$
\frac{1}{k_{0}}=\mathbf{w}^{\mathrm{T}} \mathbf{K}_{r}^{-1} \mathbf{w}-\frac{1}{k_{r}}
$$

used in [20]. Each modal frequency response curve is positive for frequencies below the corresponding modal frequency and negative for frequencies above. Thus, the quasi-static background correction (18) will lead to a misrepresentation of the contributions from modes with frequencies below the resonant frequency $\omega_{r}$ of the targeted mode. In contrast, the efficiency and robustness of the two-term correction in Eq. (12) has been demonstrated in [22] for mechanical tuned mass and inerter-based dampers.

\subsection{Force and impedance relations}

Now the two residual mode correction terms are combined with the electronic components of the piezoelectric shunt damping system shown in Fig. 2(a) to derive a design method that accounts for the non-resonant modes. The transducer displacement $u$ in the force-displacement relation (6) is now eliminated by substitution into the extended single-mode representation (11), with additional flexibility and inertia correction terms,

$$
\left(\frac{C}{\theta^{2}}+\frac{1}{\mathrm{i} \omega \theta^{2} Z(\omega)}+\frac{1}{k_{r}^{\prime}}-\frac{1}{k_{r}^{\prime \prime}} \frac{\omega_{r}^{2}}{\omega^{2}}\right) f=u_{r}
$$

As this representation is expressed in terms of flexibilities the residual mode and electrical terms are additive. The equivalent mechanical model in Fig. 2(b) is therefore extended by simply placing the background correction contributions in series, as illustrated in Fig. 3(a). The resulting displacement across the absorber terminals is the modal displacement $u_{r}$, which is decomposed into its three contributions (12), where $u$ represents the displacement associated with the first two terms in Eq. (19), while $u_{r}^{\prime}$ is the displacement across the spring with stiffness $k_{r}^{\prime}$ and correspondingly $u_{r}^{\prime \prime}$ is the displacement across the inerter with inertance $k_{r}^{\prime \prime} / \omega_{r}^{2}$. 
(a)
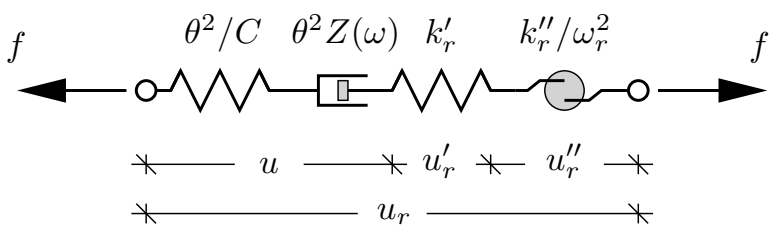

(b)

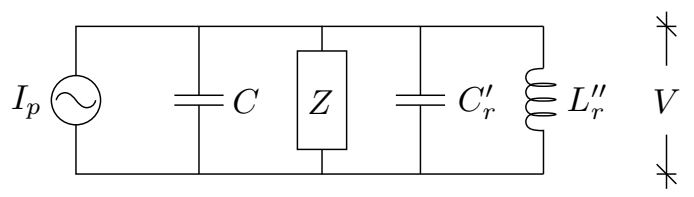

Figure 3: Equivalent (a) mechanical and (b) electronic model of piezoelectric shunt damping with additional displacements from residual mode flexibility and inertia.

The equivalent extended electronic circuit model is shown in Fig. 3(b). The two residual mode contributions correspond to a capacitance and an inductance

$$
C_{r}^{\prime}=\frac{\theta^{2}}{k_{r}^{\prime}} \quad, \quad L_{r}^{\prime \prime}=\frac{k_{r}^{\prime \prime}}{\omega_{r}^{2} \theta^{2}}
$$

placed in parallel with the actual electronic components in Fig. 2(a). For vanishing inertia correction $\left(1 / k_{r}^{\prime \prime}=0\right)$ the corresponding inductance $L_{r}^{\prime \prime} \rightarrow \infty$, corresponding to a model with a quasi-static background mode correction. If furthermore the flexibility correction is negligible $\left(1 / k_{r}^{\prime}=0\right)$, the residual mode capacitance $C_{r}^{\prime}=0$. This corresponds to blocking the current through the respective branches in Fig. 3(b), thus reverting to the original shunt circuit model in Fig. 2(a).

From the equivalent electronic model in Fig. 3(b) it follows directly that the two capacitances $C$ and $C_{r}^{\prime}$ placed in parallel can be merged to a single modified modal capacitance

$$
C_{r}=C+C_{r}^{\prime}=\left(1+\kappa_{r}^{\prime}\right) C
$$

introducing the correction factor

$$
\kappa_{r}^{\prime}=\frac{C_{r}^{\prime}}{C}=\frac{\theta^{2}}{C k_{r}^{\prime}}
$$

in which $1 / k_{r}^{\prime}$ defined by Eq. (16) accounts for the quasi-static flexibility from the residual vibration modes. Thus, for negligible residual mode flexibility $\left(1 / k_{r}^{\prime}=0\right)$ the correction factor $\kappa_{r}^{\prime}=0$ and the modal capacitance $C_{r}$ in Eq. (21) consistently recovers the actual transducer capacitance $C$.

When introducing the modal capacitance $C_{r}$ defined in Eq. (21) the modal flexibility relation (19) reduces to

$$
\left(\frac{C_{r}}{\theta^{2}}+\frac{1}{\mathrm{i} \omega \theta^{2} Z(\omega)}-\frac{1}{\omega^{2} \theta^{2} L_{r}^{\prime \prime}}\right) f=u_{r}
$$

The remaining inductance $L_{r}^{\prime \prime}$ in Fig. $3(\mathrm{~b})$ is now absorbed by the shunt impedance $Z(\omega)$ to constitute a representative modal impedance $Z_{r}(\omega)$. Because $Z(\omega)$ and $L_{r}^{\prime \prime}$ are placed in parallel 
(a)

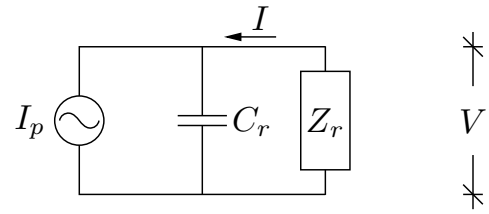

(b)

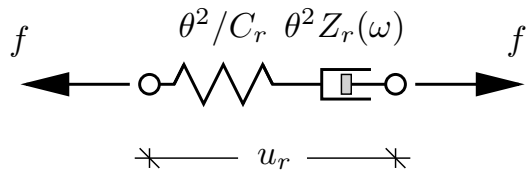

Figure 4: (a) Piezoelectric transducer with modified capacitance $C_{r}$ connected to electronic shunt circuit with modified impedance $Z_{r}(\omega)$ and (b) equivalent mechanical model.

the modal impedance is defined via the reciprocal of the individual components,

$$
\frac{1}{Z_{r}(\omega)}=\frac{1}{Z(\omega)}+\frac{1}{\mathrm{i} \omega L_{r}^{\prime \prime}}
$$

which determines the modal impedance as

$$
Z_{r}(\omega)=\frac{\mathrm{i} \omega L_{r}^{\prime \prime} Z(\omega)}{\mathrm{i} \omega L_{r}^{\prime \prime}+Z(\omega)}
$$

This modal impedance function defines the corrected shunt circuit relation $V=Z_{r}(\omega) I$, which takes into account the inertia correction from residual modes of the flexible structure. In the case of a negligible inertia contribution from the residual vibration modes the inductance $L_{r}^{\prime \prime} \rightarrow \infty$ and the modal impedance $Z_{r}(\omega)$ retains the original impedance function $Z(\omega)$ with the actual resistance $R$ and inductance $L$.

By the introduction of the modal capacitance $C_{r}$ in Eq. (21) and the modal shunt circuit impedance $Z_{r}(\omega)$ in Eq. (25), the format of the original shunt circuit model in Fig. 2 is now reestablished, as illustrated by the modal electronic model of the piezoelectric shunt damping concept in Fig. 4(a). For the corresponding mechanical model in Fig. 4(b), with modal displacement $u_{r}$ across the transducer, the modal stiffness and viscous coefficient are given as $\theta^{2} / C_{r}$ and $\theta^{2} Z_{r}(\omega)$, respectively. Therefore, the modal force-displacement relation is similar to Eq. (6) and when normalized to unit stiffness it can be written as

$$
u_{r}=\left(1+\frac{1}{\mathrm{i} \omega C_{r} Z_{r}(\omega)}\right) \frac{f}{\kappa_{r} k_{r}}
$$

with the modal electromechanical coupling coefficient $\kappa_{r}$ defined as

$$
\kappa_{r}=\frac{\theta^{2}}{C_{r} k_{r}}=\frac{\theta^{2}}{C k_{r}} \frac{1}{1+\kappa_{r}^{\prime}}
$$

In the case of negligible residual mode flexibility $\left(\kappa_{r}^{\prime}=0\right)$ this modal coupling coefficient recovers the expression $\kappa_{r}=\theta^{2} /\left(C k_{r}\right)$ for an ideal single-mode structure without any spill-over from residual vibration forms. 


\section{Calibration procedure}

This section considers a procedure for calibrating series and parallel $R L$ shunt circuit components, explicitly compensating for the influence from residual vibration modes. As demonstrated in Fig. 4 the quasi-static correction has been absorbed by the modal capacitance $C_{r}$ in Eq. (21), while the inertia correction, represented by the inductance $L_{r}^{\prime \prime}$, is combined with $Z(\omega)$ to form the modal impedance $Z_{r}(\omega)$ in Eq. (25). With the purpose of accurate calibration of the resonant shunt circuits an equivalent single-mode model is constructed, where modified shunt circuit components $R_{r}$ and $L_{r}$ contain the residual mode correction coefficients $\kappa_{r}^{\prime}$ and $\kappa_{r}^{\prime \prime}$. Because of this explicit correction, any preferred design procedure or criteria, providing optimal values for the shunt circuit resistance and inductance, can in fact be corrected by the present approach, resulting in the desired damping performance for transducers acting on flexible structures with significant modal spill-over from non-resonant modes. The influence of the residual mode corrections is illustrated by a numerical example in Section 5 .

\subsection{Series shunt circuit}

Traditionally, the series $R L$ shunt circuit is considered the primary configuration, as it was originally proposed in [4]. For the series connection the shunt circuit impedance, representing the relation in (5) between voltage $V$ and current $I$, is given by the sum of the impedances from the individual components,

$$
Z(\omega)=R+\mathrm{i} \omega L
$$

The corresponding modal impedance function $Z_{r}(\omega)$ is obtained by substitution of Eq. (28) into Eq. (25),

$$
Z_{r}(\omega)=\frac{\mathrm{i} \omega L_{r}^{\prime \prime}(R+\mathrm{i} \omega L)}{\mathrm{i} \omega L_{r}^{\prime \prime}+(R+\mathrm{i} \omega L)}=\frac{\mathrm{i} \omega L_{r}^{\prime \prime}\left(R^{2}+\omega^{2} L\left(L+L_{r}^{\prime \prime}\right)-\mathrm{i} \omega R L^{\prime \prime}\right)}{R^{2}+\omega^{2}\left(L+L_{r}^{\prime \prime}\right)^{2}}
$$

where the latter representation separates the real and the imaginary part. For vanishing inertia correction $L_{r}^{\prime \prime} \rightarrow \infty$, whereby $Z_{r}(\omega)$ recovers $Z(\omega)$ in Eq. (28). For finite values of $L_{r}^{\prime \prime}$ an approximate additive format of Eq. (29) is obtained for calibration purposes by using that in series $R L$ shunt circuits the resistive term is much smaller than the corresponding inductive contribution, as only a small phase shift of the shunt circuit resonance is required to obtain the desired resonant attenuation. Thus, for all practical purposes $R \ll \omega L$ around the resonant frequency $\omega \simeq \omega_{r}$. Hereby the two terms in Eq. (29) with $R^{2}$ may be omitted for calibration purposes. With this approximation the desired additive impedance format is then recovered,

$$
Z_{r}(\omega)=R_{r}+\mathrm{i} \omega L_{r}
$$

with modal inductance $L_{r}$ and resistance $R_{r}$ defined as

$$
L_{r}=\frac{L}{1+L / L_{r}^{\prime \prime}} \quad, \quad R_{r}=\frac{R}{\left(1+L / L_{r}^{\prime \prime}\right)^{2}}
$$


(a)

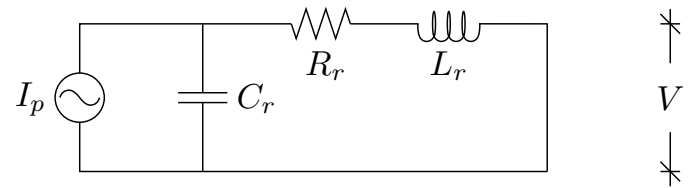

(b)

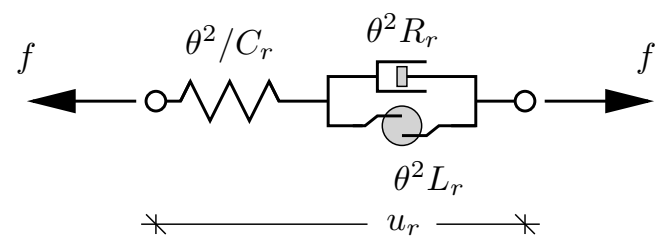

Figure 5: Equivalent (a) electronic and (b) mechanical models of series $R L$ shunt damping.

These modifications are similar to those obtained in [22] for the mechanical stiffness and damping of tuned mass or inerter dampers.

Calibration expressions for shunt circuits are commonly presented in non-dimensional form by normalization with the capacitance and resonance frequency. When normalized by $C_{r}$ and $\omega_{r}$ the expressions in (31) can be written as

$$
\lambda_{r}=L_{r} C_{r} \omega_{r}^{2}=\frac{L C_{r} \omega_{r}^{2}}{1+\kappa_{r}^{\prime \prime} L C_{r} \omega_{r}^{2}} \quad, \quad \rho_{r}=R_{r} C_{r} \omega_{r}=\frac{R C_{r} \omega_{r}}{\left(1+\kappa_{r}^{\prime \prime} L C_{r} \omega_{r}^{2}\right)^{2}}
$$

where $\lambda_{r}$ and $\rho_{r}$ represent the normalized inductance and resistance in the equivalent single-mode model in Fig. 5, while

$$
\kappa_{r}^{\prime \prime}=\frac{\theta^{2}}{C_{r} k_{r}^{\prime \prime}}=\kappa_{r} \frac{k_{r}}{k_{r}^{\prime \prime}}
$$

represents the inertia correction from residual mode spill-over.

The influence from residual vibration modes is now represented by the modified parameters $C_{r}, R_{r}$ and $L_{r}$. Thus, available calibration formulae for the series shunt circuit components can be applied for $\lambda_{r}=L_{r} C_{r} \omega_{r}^{2}$ and $\rho_{r}=R_{r} C_{r} \omega_{r}$, whereby inversion of the expressions in (32) provides the corrected values for the actual inductance $L$ and resistance $R$. The inversion of Eq. (32a) gives the optimal shunt circuit inductance as

$$
L C \omega_{r}^{2}=\frac{\lambda_{r}}{\left(1+\kappa_{r}^{\prime}\right)\left(1-\kappa_{r}^{\prime \prime} \lambda_{r}\right)}
$$

while the corresponding resistance is subsequently determined by Eq. (32b),

$$
R C \omega_{r}=\frac{\rho_{r}}{\left(1+\kappa_{r}^{\prime}\right)\left(1-\kappa_{r}^{\prime \prime} \lambda_{r}\right)^{2}}
$$

The optimal expressions for $\lambda_{r}$ and $\rho_{r}$ can now be derived for an ideal single-mode structure or taken from any set of calibration expressions proposed in the vast literature concerning piezoelectric $R L$ shunt damping, see for example $[4,5,6,9,10,11,12,13,14]$. Table 1 summarizes some of the most basic design formulae for $\lambda_{r}$ and $\rho_{r}$. 
Table 1: Single-mode calibration of series $R L$ shunt.

\begin{tabular}{lcc}
\hline & $\lambda_{r}$ & $\rho_{r}$ \\
\hline Maximum damping [4] & $\frac{1}{\left(1+\kappa_{r}\right)^{2}}$ & $\sqrt{\frac{4 \kappa_{r}}{\left(1+\kappa_{r}\right)^{3}}}$ \\
Minimum amplitude [4] & $\frac{1}{1+\kappa_{r}}$ & $\sqrt{\frac{2 \kappa_{r}}{\left(1+\kappa_{r}\right)^{2}}}$ \\
Balanced calibration [14] & $\frac{1}{\left(1+\kappa_{r}\right)^{2}}$ & $\sqrt{\frac{2 \kappa_{r}}{\left(1+\kappa_{r}\right)^{3}}}$ \\
\hline
\end{tabular}

By a pole placement principle the maximum damping is readily obtained at the bifurcation point of the two complex poles of the targeted vibration form. Alternatively, the frequency response amplitude can be minimized by leveling the vibration amplitudes at the two neutral points of the frequency response curve, at which the amplitude is independent of the resistance, determined subsequently by also leveling the amplitude at the resonance frequency. The maximum damping principle and the minimum amplitude approach based on neutral point calibration are both proposed in [4] and summarized in Table 1. However, as also demonstrated in [4] the maximum damping calibration yields an undesirable peak in the frequency amplitude due to constructive interference between the two double-root modes, while the minimum amplitude calibration results in reduced damping because of a detuning of the shunt circuit inductance.

As demonstrated in $[14,20]$ a balanced calibration principle constitutes a compromise between large attainable damping and effective response reduction. The balanced calibration principle was proposed for mechanical tuned mass dampers in [8]. It is based on equal damping of the two modes associated with the resonant vibration form of the structure. The desired flat plateau in the dynamic amplification curve is obtained at a damping level of a factor $\sqrt{2}$ below the bifurcation value used in the maximum damping principle, thus avoiding the constructive modal interference inherently associated with double-roots in the characteristic equation. For other resonant formats considered in [31] the dynamic amplification curve obtained by the balanced calibration is not ideally flat. But as equal modal damping is a robust optimality criterion, independent of the particular loading scenario, a slight inclination in the response curves is often acceptable. The balanced calibration principle has been applied for piezoelectric $R L$ shunt damping in [14, 20], without the present correction for residual mode flexibility and inertia.

The expressions in Table 1 show that the balanced calibration for the series $R L$ shunt actually combines the inductance calibration of the maximum damping approach obtained by a resistance tuning that, for small values of $\kappa_{r}$, is practically identical to the result of the minimum amplitude principle. Note, that the resistance $\rho_{r}$ obtained by the balanced calibration is, as just explained, a factor $\sqrt{2}$ smaller than the value from the maximum damping principle associated with the bifurcation point of the complex poles. 


\subsection{Parallel shunt circuit}

In case of the parallel $R L$ shunt circuit the impedance function is conveniently formulated in terms of the inverse of the individual contributions,

$$
\frac{1}{Z(\omega)}=\frac{1}{R}+\frac{1}{\mathrm{i} \omega L}
$$

whereby the impedance function can be written as

$$
Z(\omega)=\frac{\mathrm{i} \omega L R}{\mathrm{i} \omega L+R}
$$

Substitution into the expression (25) for the modal impedance function gives directly the desired form similar to Eq. (37),

$$
Z_{r}(\omega)=\frac{\mathrm{i} \omega L_{r} R}{\mathrm{i} \omega L_{r}+R}
$$

with the modal inductance $L_{r}$ defined in Eq. (31). Because $L$ and $L_{r}^{\prime \prime}$ are placed in parallel the inertia correction is absorbed directly by $L_{r}$ and the modal parallel $R L$ shunt circuit in Fig. 6(a) is therefore obtained without any approximations associated with small shunt circuit damping.

The optimal calibration formulae are derived for the non-dimensional inductance $\lambda_{r}=L_{r} C_{r} \omega_{r}^{2}$ and resistance $\rho_{r}=R C_{r} \omega_{r}$, where the actual resistance $R$ appears explicitly because it is not directly affected by the residual mode corrections. Table 2 presents the tuning expressions for the non-dimensional components based on the principles of maximum damping [10] and minimum vibration amplitude [5], and based on the balanced calibration with equal modal damping [14]. The actual shunt circuit inductance is then obtained by an expression identical to Eq. (34)

$$
L C \omega_{r}^{2}=\frac{\lambda_{r}}{\left(1+\kappa_{r}^{\prime}\right)\left(1-\kappa_{r}^{\prime \prime} \lambda_{r}\right)}
$$

The calibration of the resistance $R$ is only modified by the residual mode flexibility via the modal capacitance $C_{r}$, whereby the corresponding resistance formula appears as

$$
R C \omega_{r}=\frac{\rho_{r}}{\left(1+\kappa_{r}^{\prime}\right)}
$$

(a)

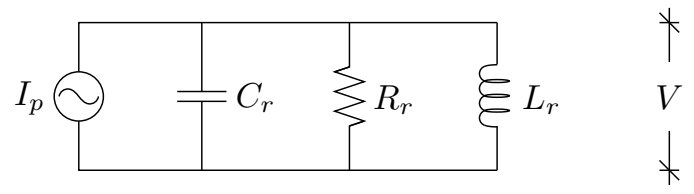

(b)

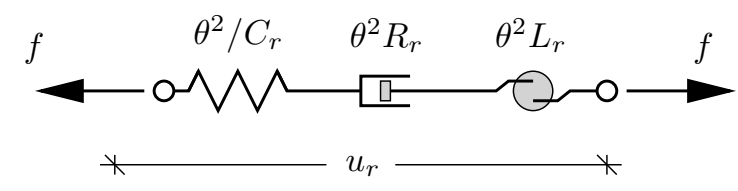

Figure 6: Equivalent electronic models of parallel $R L$ shunt damping. 


\begin{tabular}{|c|c|c|}
\hline & $\lambda_{r}$ & $\rho_{r}$ \\
\hline Maximum damping [10] & 1 & $\frac{1}{4 k}$ \\
\hline \multirow{2}{*}{ Minimum amplitude [5] } & 1 & 1 \\
\hline & $\overline{1-\kappa_{r} / 2}$ & $\overline{2 \kappa_{r}}$ \\
\hline Balanced calibration $[14]$ & 1 & $\frac{1}{9 \kappa}$ \\
\hline
\end{tabular}

without any dependence on the modal inductance $\lambda_{r}$ and the inertia correction $\kappa_{r}^{\prime \prime}$. The parallel network in Fig. 6(a) corresponds to the equivalent mechanical model in Fig. 6(b) with spring $\left(\theta^{2} / C_{r}\right)$, damper $\left(\theta^{2} R_{r}\right)$ and inerter $\left(\theta^{2} L_{r}\right)$ placed in series.

\section{Numerical example}

This example section illustrates the importance of including the residual mode flexibility and inertia correction parameters $\kappa_{r}^{\prime}$ and $\kappa_{r}^{\prime \prime}$ in the calibration of the $R L$ shunt circuit components. For the cantilever in Fig. 1 the laminate transducer acts on the deformation (curvature) of the beam, and as for the inerter-based absorber in [22] this implies that the residual mode correction is entirely dominated by the flexibility factor $\kappa_{r}^{\prime}$. Therefore, the vibrating structure is instead represented by the two-dimensional beam in Fig. 7 with length $\ell$, mass per unit length $\rho A$ and bending stiffness EI. The beam has a simple (pinned) support preventing transverse displacements at the left boundary at $x=0$, while a piezoelectric stack-actuator with axial stiffness $k_{p}$, electromechanical coupling coefficient $\theta$ and capacitance $C$ provides transverse support at the right end in $x=\ell$. This particular structure is chosen as illustration because its low-frequency modes are mainly affected by the flexibility correction $\kappa_{r}^{\prime}$, while the influence of the corresponding inertia correction $\kappa_{r}^{\prime \prime}$ increases for higher modes with more pronounced motion locally at the transducer position.

The beam is modeled by Bernoulli elements using cubic Hermitian interpolation with transverse displacement $y_{i}$ and cross section rotation $\varphi_{i}$ as the two degrees of freedom of node $i$. The corresponding mass matrix is of consistent type with element inertia distributed by the cubic shape functions. Since the stack-transducer supports the transverse displacements at the right

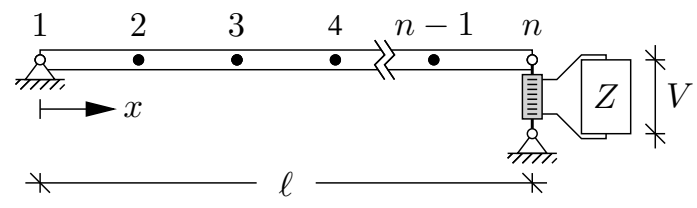

Figure 7: Discretized beam with stack transducer at the right end. 
end of the beam the connectivity vector becomes

$$
\mathbf{w}=[0, \ldots 0,1,0]^{\mathrm{T}}
$$

whereby the transducer displacement $u=\mathbf{w}^{\mathrm{T}} \mathbf{u}=y_{n}$. The resulting stiffness matrix with shortcircuit transducer electrodes is of the form

$$
\mathbf{K}=\mathbf{K}_{0}+k_{p} \mathbf{w} \mathbf{w}^{\mathrm{T}}
$$

where $\mathbf{K}_{0}$ is the stiffness matrix of the beam without the transducer attached. A simply supported beam with two pinned boundaries is recovered in the limit $k_{p} \rightarrow \infty$.

\subsection{Modal properties}

The mode shape vector $\mathbf{u}_{j}$ and natural frequency $\omega_{j}$ of the discretized beam structure with short-circuit transducer conditions can be found by solving the eigenvalue problem in Eq. (7). The solution of the underlying continuous beam problem is obtained by solving the corresponding boundary value problem with an equivalent spring support at the right end. This leads to the following expression for the mode shapes

$$
u_{r}(s)=\frac{1}{2}\left(\frac{\sin (\beta s)}{\sin (\beta)}+\frac{\sinh (\beta s)}{\sinh (\beta)}\right)
$$
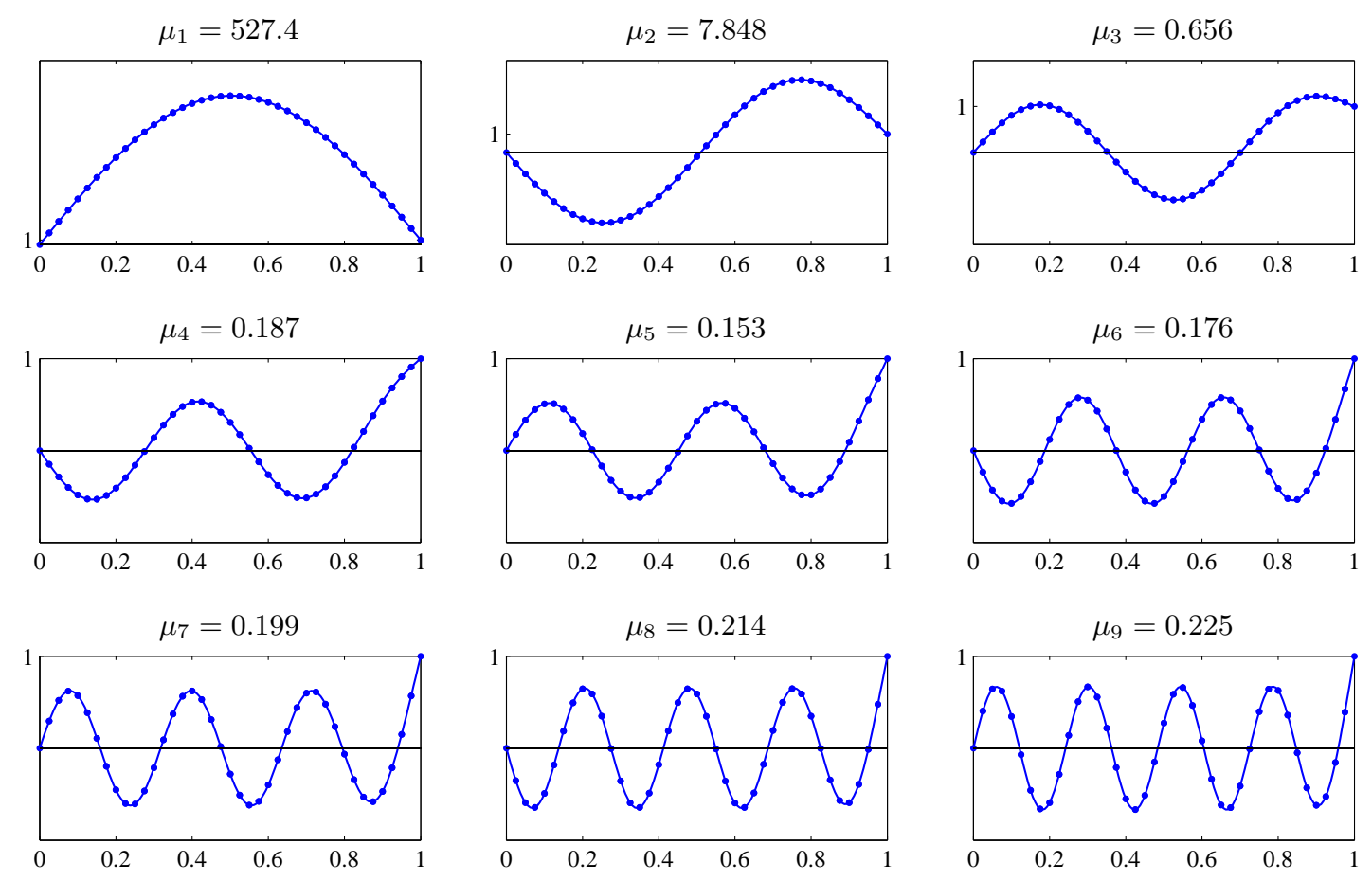

Figure 8: Modes 1 to 9 of structure (short-circuit electrodes) and $\mu_{j}=m_{j} /(\rho A \ell)$ representing relative modal mass. 
Table 3: Natural frequencies $\omega_{j} / \omega_{s}$ normalized by $\omega_{s}=(\pi / \ell)^{2} \sqrt{E I / \rho A}$.

\begin{tabular}{cccccccccc}
\hline Mode & 1 & 2 & 3 & 4 & 5 & 6 & 7 & 8 & 9 \\
\hline Discretized & 0.9901 & 3.8349 & 8.0646 & 13.049 & 19.545 & 28.451 & 39.652 & 52.987 & 68.389 \\
Exact & 0.9901 & 3.8349 & 8.0646 & 13.049 & 19.545 & 28.451 & 39.651 & 52.983 & 68.380 \\
\hline
\end{tabular}

with normalized coordinate $s=x / \ell$ and the non-dimensional wave-number $\beta$ obtained as the solution to the following transcendental equation,

$$
\tan (\beta)=\frac{\tanh (\beta)}{1-2 \nu \tanh (\beta) / \beta^{3}}
$$

where the parameter

$$
\nu=\frac{k_{p} \ell^{3}}{E I}
$$

represents the relative transducer stiffness.

Figure 8 shows the first nine mode shapes of the beam with a pinned left support and a shortcircuit piezoelectric stack-transducer, with non-dimensional stiffness $\nu=1000$, supporting the right end transversely. The mode shapes are normalized to unit displacement at the location of the transducer. Red dashed curves represent the analytical solution (43), while blue solid curves show the mode shape of the discretized beam model. Furthermore, the dots represent the displacement at the 41 nodes used in the 40 element model of the beam. The red dashed and the solid blue curves coincide, which validates the particular element discretization. This is furthermore confirmed by the corresponding natural frequencies $\omega_{j}$ in Table 3 , where a relative deviation of less than $10^{-3}$ is observed for mode 9. In Fig. 8 the relative modal mass $\mu_{j}=m_{j} /(\rho A \ell)$ identifies the ratio between modal mass and the total translational inertia of the beam. It is seen that minimum relative modal mass occurs for mode 5 , for which the authority of the transducer with $\nu=1000$ is therefore optimal. For the low-frequency modes $(j<5)$ the authority of the actuator is limited because of the small displacement at transducer location at the right end of beam, while in the high-frequency limit $(j>5)$ it is increasingly localized around the boundary and consequently reduced. As demonstrated next, effective resonant damping of the vibration modes with limited transducer authority is obtained by the proposed calibration formulae with explicit residual mode correction.

\subsection{Complex mode analysis}

The free vibration properties of the damped structure are investigated by solving the eigenvalue problem for the beam structure with the stack transducer shunted to a series or parallel $R L$ network. The frequency equations (1) and (6) are written in a compact state-space format, whereby the complex-valued natural frequencies $\omega_{j}$ can be derived from the eigenvalues to the corresponding 
Table 4: Design of piezoelectric $R L$ shunt damping.

\begin{tabular}{lll}
\hline Transducer design & & \\
\hline Desired damping ratio: & $\zeta_{d}$ & \\
Coupling parameter: & $\kappa_{r}=8 \zeta_{d}^{2}$ & \\
Correction parameters: & $\kappa_{r}^{\prime}=\frac{\kappa_{r}\left(k_{r} / k_{r}^{\prime}\right)}{1-\kappa_{r}\left(k_{r} / k_{r}^{\prime}\right)}$, & $\kappa_{r}^{\prime \prime}=\kappa_{r}\left(k_{r} / k_{r}^{\prime \prime}\right)$ \\
Transducer parameter: & $\frac{\theta^{2}}{C}=\kappa_{r} k_{r}\left(1+\kappa_{r}^{\prime}\right)$ & \\
\hline Shunt circuit calibration & Series & Parallel \\
\hline Single-mode parameters: & $\frac{1}{\left(1+\kappa_{r}\right)^{2}}$ & 1 \\
$\lambda_{r}=$ & $\sqrt{\frac{2 \kappa_{r}}{\left(1+\kappa_{r}\right)^{3}}}$ & $\sqrt{\frac{1}{2 \kappa_{r}}}$ \\
$\rho_{r}=$ & $\frac{\lambda_{r}}{\left(1+\kappa_{r}^{\prime}\right)\left(1-\kappa_{r}^{\prime \prime} \lambda_{r}\right)}$ & $\frac{\rho_{r}}{\left(1+\kappa_{r}^{\prime}\right)\left(1-\kappa_{r}^{\prime \prime} \lambda_{r}\right)}$ \\
Shunt circuit parameters: & $\frac{\rho_{r}}{\left(1+\kappa_{r}^{\prime}\right)\left(1-\kappa_{r}^{\prime \prime} \lambda_{r}\right)^{2}}$ & $\frac{1}{2+\kappa_{r}^{\prime}}$ \\
$L C \omega_{r}^{2}=$ &
\end{tabular}

state-space matrix, while the damping ratios are subsequently determined as the relative imaginary part by the relation $\zeta_{j}=\operatorname{Im}\left[\omega_{j}\right] /\left|\omega_{j}\right|$.

The $R L$ circuit components are calibrated using the balanced calibration principle with calibration formulae for the equivalent single-mode system given in the bottom rows of Table 1 (series) and Table 2 (parallel). The balanced damping principle is based on equal damping ratio of the two modes in which the original resonant mode is split by shunting with the $R L$ circuit. Furthermore, the dynamic amplification factor, $D A F$, of the resonant mode has a nearly level plateau around the resonance frequency, within which $D A F \simeq 1 /\left(2 \zeta_{r}\right)$, [8]. The dynamic amplification factor can therefore be chosen to define the desired damping ratio as $\zeta_{d}=1 /(2 D A F)$ for the targeted vibration mode $r$. This is chosen as the starting point of the calibration procedure described in Table 4. Initially the desired damping ratio defines the modal coupling parameter $\kappa_{r}=8 \zeta_{d}^{2}$, as demonstrated in detail in [20]. The flexibility ratios $k_{r} / k_{r}^{\prime}$ and $k_{r} / k_{r}^{\prime \prime}$ are then determined by the expressions in (16) and (17), respectively, whereby the flexibility coefficient $\kappa_{r}^{\prime}$ can be determined by Eq. (27), while the corresponding inertia coefficient $\kappa_{r}^{\prime \prime}$ follows directly from Eq. (33). The transducer parameter $\theta^{2} / C$ is then finally determined by Eq. (27).

The calibration procedure for the $R L$ shunt circuit components initially determines the nondimensional single-mode parameters $\lambda_{r}$ and $\rho_{r}$, according to the preferred calibration principle, which in the present case is the balanced calibration with expressions given in Tables 1 and 2 . The actual inductance $L$ and resistance $R$ are then obtained by the expressions (34) and (35) for 
the series network or (39) and (40) for the parallel version, as summarized in the two bottom rows of Table 4.

Table 5: Complex root analysis for $\zeta_{d}=0.02$ and $\kappa_{r}=0.0032$

\begin{tabular}{|c|c|c|c|c|c|c|c|c|c|}
\hline Mode & 1 & 2 & 3 & 4 & 5 & 6 & 7 & 8 & 9 \\
\hline$\kappa_{r}^{\prime}$ & 0.1934 & 0.0425 & 0.0173 & 0.0087 & 0.0109 & 0.0170 & 0.0242 & 0.0327 & 0.0427 \\
\hline$\kappa_{r}^{\prime \prime}$ & 0.0021 & 0.0049 & 0.0054 & 0.0053 & 0.0122 & 0.0229 & 0.0339 & 0.0455 & 0.0580 \\
\hline$\tilde{\kappa}_{r} / \kappa_{r}$ & 1.0021 & 1.0049 & 1.0054 & 1.0053 & 1.0124 & 1.0233 & 1.0349 & 1.0475 & 1.0614 \\
\hline \multicolumn{10}{|c|}{ Series } \\
\hline$L C \omega_{r}^{2} / \lambda_{r}$ & 0.8397 & 0.9638 & 0.9884 & 0.9966 & 1.0014 & 1.0062 & 1.0104 & 1.0142 & 1.0177 \\
\hline$R C \omega_{r} / \rho_{r}$ & 0.8414 & 0.9685 & 0.9937 & 1.0019 & 1.0137 & 1.0296 & 1.0456 & 1.0622 & 1.0800 \\
\hline & 0.0200 & 0.0200 & 0.0200 & 0.0200 & 0.0200 & 0.0200 & 0.0201 & 0.0201 & 0.0201 \\
\hline$\zeta_{r}$ & 0.0200 & 0.0200 & 0.0200 & 0.0200 & 0.0200 & 0.0200 & 0.0200 & 0.0199 & 0.0199 \\
\hline$\zeta^{0}$ & 0.0398 & 0.0283 & 0.0229 & 0.0208 & 0.0194 & 0.0181 & 0.0170 & 0.0161 & 0.0153 \\
\hline$\zeta_{r}$ & 0.0032 & 0.0122 & 0.0172 & 0.0191 & 0.0201 & 0.0209 & 0.0215 & 0.0220 & 0.0223 \\
\hline & 0.0200 & 0.0200 & 0.0201 & 0.0201 & 0.0201 & 0.0202 & 0.0204 & 0.0205 & 0.0206 \\
\hline$\zeta_{r}$ & 0.0200 & 0.0200 & 0.0200 & 0.0200 & 0.0201 & 0.0202 & 0.0204 & 0.0205 & 0.0206 \\
\hline \multicolumn{10}{|c|}{ Parallel } \\
\hline$L C \omega_{r}^{2} / \lambda_{r}$ & 0.8397 & 0.9639 & 0.9884 & 0.9966 & 1.0015 & 1.0063 & 1.0106 & 1.0145 & 1.0181 \\
\hline$R C \omega_{r} / \rho_{r}$ & 0.8379 & 0.9592 & 0.9830 & 0.9913 & 0.9892 & 0.9833 & 0.9764 & 0.9684 & 0.9590 \\
\hline & 0.0200 & 0.0200 & 0.0200 & 0.0200 & 0.0200 & 0.0200 & 0.0200 & 0.0200 & 0.0200 \\
\hline$\zeta_{r}$ & 0.0200 & 0.0200 & 0.0200 & 0.0200 & 0.0200 & 0.0200 & 0.0200 & 0.0200 & 0.0200 \\
\hline$\zeta^{0}$ & 0.0339 & 0.0270 & 0.0226 & 0.0207 & 0.0194 & 0.0181 & 0.0171 & 0.0161 & 0.0153 \\
\hline$\zeta_{r}$ & 0.0032 & 0.0121 & 0.0170 & 0.0190 & 0.0201 & 0.0211 & 0.0218 & 0.0224 & 0.0228 \\
\hline \multirow{2}{*}{$\tilde{\zeta}_{r}$} & 0.0200 & 0.0201 & 0.0201 & 0.0201 & 0.0201 & 0.0202 & 0.0203 & 0.0205 & 0.0206 \\
\hline & 0.0200 & 0.0200 & 0.0200 & 0.0200 & 0.0201 & 0.0202 & 0.0204 & 0.0205 & 0.0206 \\
\hline
\end{tabular}

In the following the transducer properties and electronic shunt circuit components are calibrated individually for the first nine vibration modes of the beam structure, with a desired damping ratio $\zeta_{d}=0.02$. Thus, the governing transducer parameters $\theta$ and $C$ vary for each resonant vibration mode $r$, representing a transducer with different properties. The results of the design procedure and the corresponding modal damping ratios are presented for both series and parallel shunt circuit components in Table 5 for the target mode from $r=1$ to 9 . It is seen that for modes 1 to 4 the flexibility correction factor $\kappa_{r}^{\prime}$ is larger than the corresponding inertia coefficient $\kappa_{r}^{\prime \prime}$, while for modes 6 and upwards the inertia correction becomes increasingly important.

In the table the optimal values of the actual resistance and inductance are presented relative to the values of $\lambda_{r}$ and $\rho_{r}$ for the idealized single-mode structure. Thus, for a damped structure without significant influence from residual vibration modes these ratios are close to unity, as it is seen to be the case for mode $r=5$, which has previously been identified as the vibration mode with best modal authority. 
For the series $R L$ circuit the actual inductance and resistance for the first modes are smaller than the ideal single-mode values because of the division by $\left(1+\kappa_{r}^{\prime}\right)$ in Eqs. (34) and (35), while for the higher modes the inertia correction becomes increasingly important and the inductance and resistance ratios therefore become larger than unity as the factor $\left(1-\kappa_{r}^{\prime \prime} \lambda_{r}\right)$ decreases correspondingly. For mode 1 both optimal components are approximately $16 \%$ smaller than for the single-mode system, while for mode 9 they are $1.8 \%$ and $8 \%$ larger, respectively.

The two damping ratios $\zeta_{r}$ for the split target modes are presented in the table in the order according to the magnitude of the associated natural frequency. For the series $R L$ circuit the desired damping ratio $\zeta_{d}=0.02$ is attained exactly for the first six modes, while insignificant deviations occur for the higher modes. The damping ratio $\zeta_{r}^{0}$ is obtained by a calibration without correction for residual mode spill-over $\left(\kappa_{r}^{\prime}=0\right.$ and $\left.\kappa_{r}^{\prime \prime}=0\right)$. In this case the damping ratio associated with the highest of the two mode 1 frequencies is reduced by almost an order of magnitude to 0.0032 , while for mode 9 the damping of the lowest frequency is $23.5 \%$ smaller than the desired value (0.02). The transition, where the smallest damping ratio shifts from the highest to the lowest of the two frequencies, occurs in this case at mode 5 with maximum authority and a damping ratio $\zeta_{r}^{0}=0.0194$ that is only $3 \%$ smaller than the targeted 0.02 .

For the parallel $R L$ circuit the relative inductance is similar to that of the series network, while the relative resistance ratio is smaller than the ratio for the series circuit and even below unity. Because approximations are not required in the calibration of the parallel shunt circuit the desired damping ratio is reproduced exactly for all reported vibration modes 1 to 9 . Thus, according to the complex root analysis the optimally calibrated parallel $R L$ shunt should indeed outperform its series counterpart, although the discrepancies are insignificant in the present example. In Section 5.3 this is further investigated by a harmonic response analysis.

The generalized electromechanical coupling coefficient is often estimated numerically or experimentally by the classic relation $[1,24,25,26]$

$$
\tilde{\kappa}_{r}=\frac{\Omega_{r}^{2}-\omega_{r}^{2}}{\omega_{r}^{2}}
$$

The open-circuit natural frequency $\Omega_{r}$ is inherently affected by spill-over from the residual modes and $\tilde{\kappa}_{r}$ may therefore be used as an accurate estimate of the modal electromechanical coupling coefficient $\kappa_{r}$. It has been demonstrated in [24] that $\tilde{\kappa}_{r}$ exactly recovers the analytical expression for generalized electromechanical coupling coefficient $\theta^{2} /\left(C k_{r}\right)$ when the explicit interaction with residual modes can be neglected. The ratio $\tilde{\kappa}_{r} / \kappa_{r}$ in Table 5 is in fact close to unity for the first couple of modes, demonstrating that $\tilde{\kappa}_{r}$ actually captures the residual mode correction represented by $\kappa_{r}^{\prime}$ in Eq. (27). However, the ratio increases gradually with $r$ as the residual mode inertia implicitly affects $\Omega_{r}^{2}$ in Eq. (46), while $\kappa_{r}$ only depends on the residual mode flexibility $\kappa_{r}^{\prime}$ and the inertia coefficient $\kappa_{r}^{\prime \prime}$ instead modifies the impedance function. 
Table 5 also presents the two damping ratios $\tilde{\zeta}_{r}$ of the two split modes obtained by using the estimate $\tilde{\kappa}_{r}$ instead of $\kappa_{r}$ to determine the single-mode shunt circuit components $\lambda_{r}$ and $\rho_{r}$. Thus, the electromechanical stiffness $\theta^{2} / C$ and the residual mode coefficients $\kappa_{r}^{\prime}$ and $\kappa_{r}^{\prime \prime}$ are identical to those in the previous analysis based on $\kappa_{r}$, and the targeted damping ratio is therefore still $\zeta_{d}=0.02$. It is seen that the use of the estimated value $\tilde{\kappa}_{r}$ also results in equal modal damping, while the minor deviations compared to the desired value $\zeta_{d}=0.02$ occur because of the observed difference between $\kappa_{r}$ and $\tilde{\kappa}_{r}$. Thus, the present calibration procedure is rather robust when the correction with respect to the residual mode coefficients $\kappa_{r}^{\prime}$ and $\kappa_{r}^{\prime \prime}$ is included in the calibration of $L$ and $R$.

\subsection{Harmonic response analysis}

As resonant absorbers are primarily aimed at reducing harmonic response amplitudes the accuracy of the proposed residual mode compensation is illustrated by a harmonic response analysis. The external load vector is defined as $\mathbf{f}_{e}=\mathbf{M} \mathbf{u}_{r} /\left(\mathbf{w}^{\mathrm{T}} \mathbf{u}_{r}\right)$, whereby the resonant modal load $f_{j}=m_{j} \delta_{j r}$ (no summation) is by construction orthogonal to all non-resonant vibration forms $(j \neq r)$.

The dynamic response is assessed in terms of the nodal rotation $\varphi_{1}$ at the left end of the beam (node 1) in Fig. 7. The vibration amplitude is normalized by the corresponding static solution $\varphi_{1}^{0}$ to determine the dynamic amplification factor $(D A F)$. The associated piezoelectric force $f=\theta V$ is normalized by the corresponding modal load $f_{r}$.

Figure 9 shows the frequency response curves for the series $R L$ shunt circuit. The left column of figures (a,c,e) shows the dynamic amplification of the left end rotation, while the right column $(\mathrm{b}, \mathrm{d}, \mathrm{f})$ presents the frequency amplitude of the force exerted on the structure by the piezoelectric transducer. The corresponding rows in Fig. 9 represent the amplitude curves when the targeted resonant mode is $r=1(\mathrm{a}, \mathrm{b}), 5(\mathrm{c}, \mathrm{d})$ or $9(\mathrm{e}, \mathrm{f})$, respectively. Thus, each row in the figure corresponds to a particular transducer and shunt circuit configuration aimed specifically at a desired damping ratio of $\zeta_{d}=0.02$. This implies that the dynamic amplification factor in the left column of the figure is $D A F \simeq 1 /\left(2 \zeta_{d}\right)=25$ around the resonance frequency, as indicated by the horizontal dashed line. As demonstrated in [8] for the mechanical tuned mass damper the particular calibration of the damper parameter by a factor $\sqrt{2}$ below the bifurcation point value implies that the frequency amplitude of the force acting on the structure retains a fully flat plateau at the resonance frequency of unit magnitude when normalized by the modal load. This is indicated by the horizontal dashed line in the right column of figures. The solid curves in Fig. 9 are obtained by the present calibration procedure with correction for residual mode spill-over, while the dashed curves correspond to the assumed single-mode calibration without correction $\left(\kappa_{r}^{\prime}=0\right.$ and $\left.\kappa_{r}^{\prime \prime}=0\right)$. 

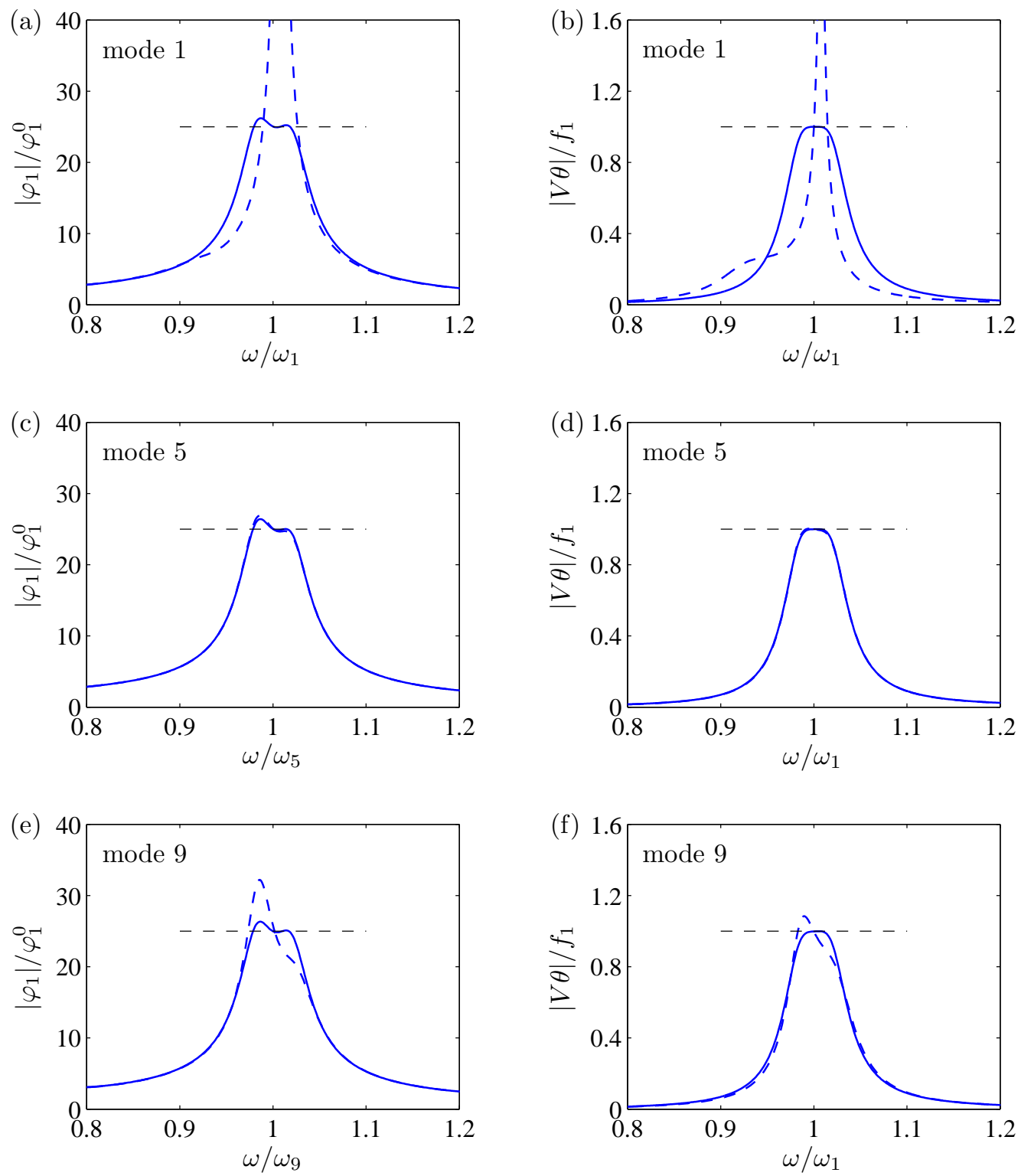

Figure 9: Series shunt: Dynamic amplification of end rotation (a,c,e) and voltage amplitude (b,d,f) for modes 1 $(\mathrm{a}, \mathrm{b}), 5(\mathrm{c}, \mathrm{d})$ and $9(\mathrm{e}, \mathrm{f})$.

In the case of resonant mode 1 excitation $(\mathrm{a}, \mathrm{b})$ the severe detuning, resulting from single-mode calibration without correction, implies that the dashed curve in the left column exhibits a very significant resonant peak with a maximum dynamic amplification of 147.8, which agrees well with $1 /(2 \cdot 0.0032)=156.3$ obtained by the damping ratio $\zeta_{1}^{0}=0.0032$ in Table 5 for the series shunt circuit. The corresponding solid curve, which represents the corrected calibration, is seen to retain the desired level of dynamic amplification as indicated by the horizontal line. For mode 5 (c,d) with large transducer authority and limited correction coefficients $\left(\kappa_{r}^{\prime}=0.0109\right.$ and $\left.\kappa_{r}^{\prime}=0.0122\right)$ the dashed and solid curves coincide, while for mode 9 (e,f) the significant inertia correction implies 

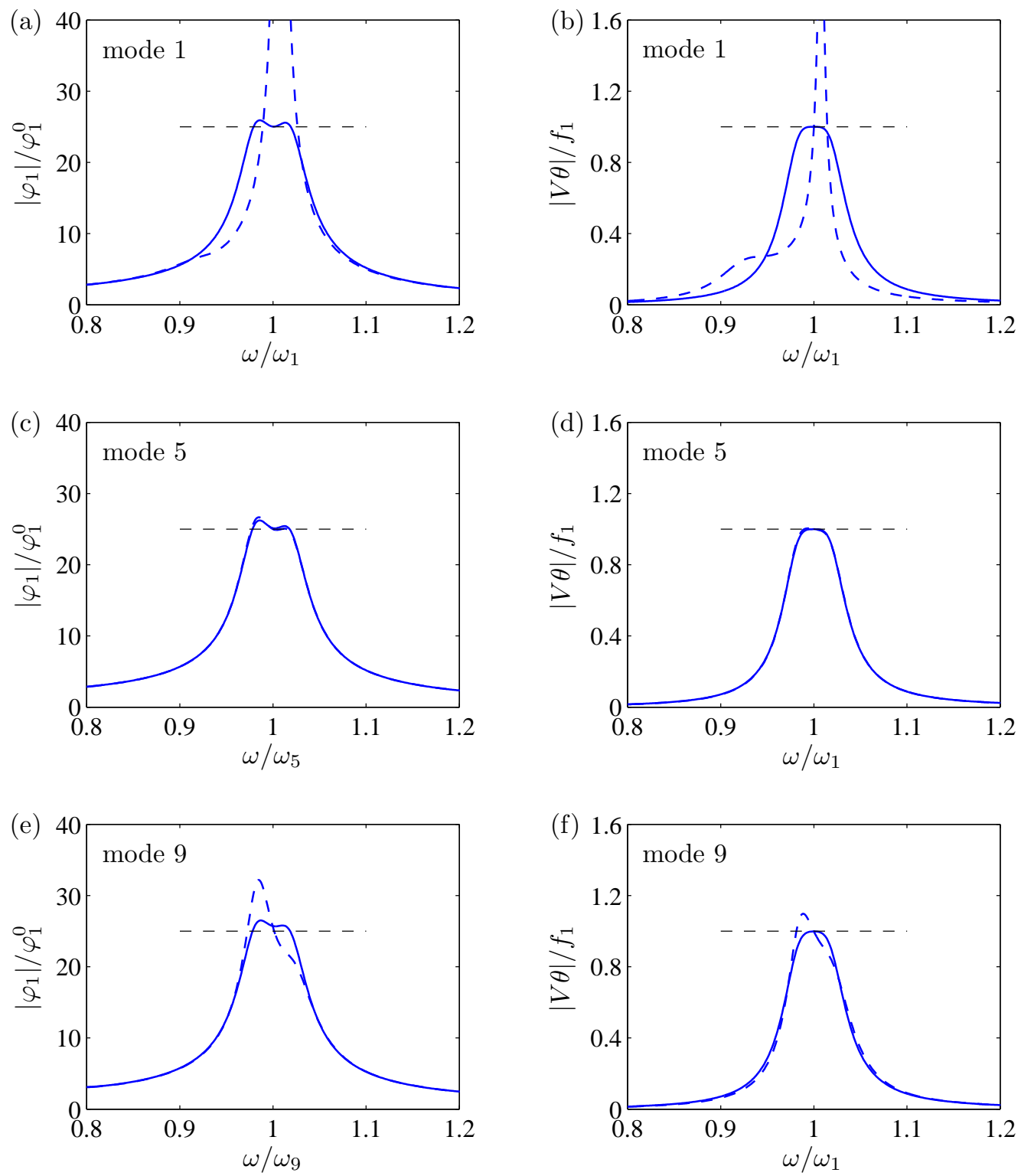

Figure 10: Parallel shunt: Dynamic amplification of end rotation (a,c,e) and voltage amplitude (b,d,f) for modes 1 $(\mathrm{a}, \mathrm{b}), 5(\mathrm{c}, \mathrm{d})$ and $9(\mathrm{e}, \mathrm{f})$.

that for the low-frequency peak with modal damping ratio $\zeta_{9}^{0}=0.0153$ an almost $30 \%$ overshoot in vibration amplitude is observed. For the piezoelectric force $f=\theta V$ shown in the right column in Fig. 9 the observations are similar to those for the dynamic amplification factor, with a severe overshoot for mode 1, a quite accurate calibration for mode 5 and a more moderate discrepancy for mode 9. Figure 10 shows the frequency amplitude curves for the parallel $R L$ shunt circuit. As for the series circuit results in Fig. 9 the solid curves for the parallel shunt circuit maintain an optimal flat plateau, without any local through and peaks. 


\section{Conclusion}

The present paper improves the calibration accuracy for piezoelectric $R L$ shunts by a consistent procedure taking into account the spill-over from the non-resonant modes of the flexible structure. The approach is based on the representation of the transducer displacement $u$ in terms of the displacement of the resonant vibration mode $u_{r}$ and two additional terms, $u_{r}^{\prime}$ and $u_{r}^{\prime \prime}$, representing the flexibility and inertia contributions from the residual vibration modes of the structure. Thus, an augmented single-mode structural model is obtained, in which the modal interaction with non-resonant modes is explicitly contained by two non-dimensional background flexibility and inertia coefficients $\kappa_{r}^{\prime}$ and $\kappa_{r}^{\prime \prime}$, which are directly available from the numerical model of the flexible structure. For piezoelectric shunt damping it is demonstrated that the residual mode flexibility is directly absorbed by the short-circuit transducer capacitance, while the inertia contribution is equivalent to a supplemental inductance and therefore conveniently included in the shunt impedance. By the generic reinterpretation of the piezoelectric shunt circuit by the residual mode contributions a pair of explicit correction formulae for the shunt circuit inductance and resistance is obtained. Thus, any set of calibration formulae for the shunt circuit components, derived on the basis of an assumed single-mode structure, can be corrected with respect to the interaction with non-resonant modes by the expressions presented in Section 4. A numerical example verifies the accurate shunt circuit calibration for an arbitrary vibration form, securing both equal damping of the targeted modes and effective response reduction by correcting the pole based balanced calibration principle to compensate for the influence from residual mode interaction. Furthermore, the numerical results indicate that the residual mode correction has a particular influence in cases where the local transducer is placed indirectly on a flexible structure. This is effectively assessed in terms of a representative modal mass ratio, such as $\mu_{j}$ in Fig. 8. For the present beam structure the modal mass is large for the first modes because the amplitude at transducer location is relatively small, whereas for higher modes it increases as the authority of the transducer is gradually limited due to the increased waviness of the mode shapes.

\section{References}

[1] A. Preumont, Vibration Control of Active Structures. An Introduction, Springer, Heidelberg, 2011.

[2] S.O.R. Moheimani, A.J. Fleming, Piezoelectric transducers for vibration control and damping, Springer, London, 2006.

[3] R.L. Forward, Electronic damping of vibrations in optical structures, Applied Optics 18 (1979) 690697.

[4] N.W. Hagood, A. von Flotow, Damping of structural vibrations with piezoelectric materials and passive electrical networks, Journal of Sound and Vibration 146 (1991) 243-268. 
[5] S.Y. Wu, Piezoelectric shunts with a parallel R-L circuit for structural damping and vibration control, Proceedings of the Society of Photo-Optical Instrumentation Engineers (SPIE) 2720, San Diego, California, February 1996, pp. 259-269.

[6] C.H. Park, D.J. Inman, A uniform model for series R-L and parallel R-L shunt circuits and power consumption, Proceedings of the Society of Photo-Optical Instrumentation Engineers (SPIE) 3668, Newport Beach, California, March 1999, pp. 797-804.

[7] J.P. Den Hartog, Mechanical Vibrations, Dover Publications, New York, 1956.

[8] S. Krenk, Frequency analysis of the tuned mass damper, Journal of Applied Mechanics 72 (2005) 936-942.

[9] J.J. Hollkamp, Multimodal passive vibration suppression with piezoelectric materials and resonant shunts, Journal of Intelligent Material Systems and Structures 5 (1994) 49-57.

[10] G. Caruso, A critical analysis of electric shunt circuits employed in piezoelectric passive vibration damping, Smart Materials and Structures 10 (2001) 1059-1068.

[11] K. Yamada, H. Matsuhisa, H. Utsuno, K. Sawada, Optimum tuning of series and parallel LR circuits for passive vibration suppression using piezoelectric elements, Journal of Sound and Vibration 329 (2010) 5036-5057.

[12] M.V. Kozlowski, D.G. Cole, R.L. Clark, A comprehensive study of the RL series resonant shunted piezoelectric: A feedback controls perspective, Journal of Vibration and Acoustics 133 (2011) 011012 (10pp).

[13] P. Soltani, G. Kerschen, G. Tondreau, A. Deraemaeker, Piezoelectric vibration damping using resonant shunt circuits: an exact solution, Smart Materials and Structures 23 (2014) 125014 (11pp).

[14] J. Høgsberg, S. Krenk, Balanced calibration of resonant shunt cicuits for piezoelectric vibration control, Journal of Intelligent Material Systems and Structures 23 (2012) 1937-1948.

[15] J.F. Deü, W. Larbi, R. Ohayon, R. Sampaio, Piezoelectric shunt vibration damping of structuralacoustic systems: Finite element formulation and reduced-order model, Journal of Vibration and Acoustics 136 (2014) 031007 (8pp).

[16] R.L. Clark, Accounting for out-of-bndwidth modes in the assumed modes approach: Implications on colocated output feedback control, Journal of Dynamic Systems, Measurement, and Control 119 (1997) 390-395.

[17] S.O.R. Moheimani, Minimizing the effect of out of bandwidth modes in truncated structure models, Journal of Dynamic Systems, Measurement, and Control 122 (2000) 237-239.

[18] O.E. Hansteen, K. Bell, Accuracy of mode superposition analysis in structural dynamics, Earthquake Engineering and Structural Dynamics 7 (1979) 405-411. 
[19] S. Krenk, J. Høgsberg, Tuned mass absorber on a flexible structure, Journal of Sound and Vibration 333 (2014) 1577-1595.

[20] J. Høgsberg, S. Krenk, Balanced calibration of resonant piezoelectric RL shunts with quasi-static background flexibility correction, Journal of Sound and Vibration 341 (2015) 16-30.

[21] M. Krommer, M. Pieber, Y. Vetyukov, Modeling, simulation and vibration reduction of thin shells with piezoelectric transducers, Elektrotechnik und Informationstechnik 132 (2015) 437-447.

[22] S. Krenk, J. Høgsberg, Tuned resonant mass or inerter-based absorbers: unified calibration with quasi-dynamic flexibility and inertia correction, Proceedings of the Royal Society A-Mathematical, Physical and Engineering Sciences 472 (2015) 20150718 (23pp).

[23] J. Ducarne, O. Thomas, J.F. Deü, Placement and dimension optimization of shunted piezoelectric patches for vibration reduction, Journal of Sound and Vibration 331 (2012) 3286-3303.

[24] O. Thomas, J.F. Deü, J. Ducarne, Vibrations of an elastic structure with shunted piezoelectric patches: efficient finite element formulation and electromechanical coupling coefficients, International Journal for Numerical Methods in Engineering 80 (2009) 235-268.

[25] J. Becker, O. Fein, M. Maess, L. Gaul, Finite element-based analysis of shunted piezoelectric structures for vibration damping, Computers and Structures 84 (2006) 2340-2350.

[26] T. Delpero, A.E. Bergamini, P. Ermanni, Identification of electromechanical parameters in piezoelectric shunt damping and loss factor prediction, Journal of Intelligent Material Systems and Structures 24 (2012) 287-298.

[27] E.H. Anderson, N.W. Hagood, Simultaneous piezoelectric sensing/actuation: Analysis and application to controlled structures, Journal of Sound and Vibration 174 (1994) 617-639.

[28] H.H. Law, P.L. Rossiter, G.P. Simon, L.L. Koss, Characterization of mechanical vibration damping by piezoelectric materials, Journal of Sound and Vibration 197 (1996) 489-513.

[29] M.C. Smith, Synthesis of mechanical networks: The inerter, IEEE/ASME Transactions on Automatic Control 47 (2002) 1648-1662.

[30] M.Z.Q. Chen, C. Papageorgiou, F. Scheibe, F.C. Wang, M.C. Smith, The missing mechanical circuit element, IEEE Circuits and systems Magazine 9 (2009) 10-26.

[31] S. Krenk, J. Høgsberg, Equal modal damping design for a family of resonant vibration control formats, Journal of Vibration and Control 19 (2013) 1294-1315. 